\title{
Review of a Predator-Prey Model with Two Limit Cycles
}

\author{
Q van der Hoff, A Harding \\ Department of Mathematics and Applied Mathematics \\ University of Pretoria \\ quay.vanderhoff@up.ac.za
}

\begin{abstract}
It is well-known that the Lotka-Volterra predator-prey model has a family of periodic orbits, but does not possess limit cycles and therefore the model is said to be structurally unstable. The Lotka-Volterra model is a special case of a much larger group namely the quadratic population models and it can be shown that none of them can produce limit cycles. The surprising finding is that by combining two quadratic models a quadratic population model with two limit cycles is uncovered. Although the model looks simple at first glance it provides a rich source of dynamics and deserves attention.

In this paper we revisit a model that has its origin in the work of Dubois and Closset [1]. A set of two quadratic population models interact as piecewise defined differential equations. The model has been discussed by Ren Yongtai and Han Li [2], cryptically written and showing some linguistic and typographical errors, but providing an excellent vehicle for developing skills in mathematical modeling, differential equations and technology for the young researcher.

We explore the model in clearer detail and supplement the theory with rich graphical illustration. The paper has the purpose of providing an example of how a young researcher, such as a post graduate student in biomathematics, can expand on an existing model by making use of current technology.
\end{abstract}

KEYWORDS: Quadratic population model, biomath education, limit cycles, piecewisedefined differential equations

\section{Introduction}

In a previous discussion paper Van der Hoff [4] highlighted the problems encountered by biology students not having enough mathematics and vice versa. It has become apparent that no single discipline can fully address the challenges of new frontiers in biology [5], and as such the average life science student is not exposed to enough areas in mathematics [3]. It was suggested that mathematical biology students must be encouraged to work across disciplinary boundaries. Here we propose an example that students of mathematics, biology or biomathematics might find accessible. The example stems from a paper that is not only dated but somewhat flawed and difficult to follow. We first provide background.

The paper of Yongtai and Li [2] builds on the work of Dubois and Closset [1] on the timedependent horizontal structuration of plankton populations called the patchiness effect, presented at a marine biology symposium in 1975. Interaction between phytoplankton and herbivorous zooplankton result in a predator-prey model. 
In the simplified model

$$
\begin{aligned}
& \frac{d x}{d t}=k_{1} x(1-\alpha x)-k(x) y \\
& \frac{d y}{d t}=-k_{3} y+\beta k(x) y
\end{aligned}
$$

$x(t)$ represents the phyloplankton biomass (the prey) and $y(t)$ the zooplankton biomass (the predator). The term $k(x)$ is called the grazing function. Dubois and Closset [1] present numerical simulations of the predator-prey models, varying the grazing rate. Small changes in the shape of the grazing curve lead to drastic differences in the plankton dynamics. Conditions are given for which there is (i) one stable stationary state, (ii) one stable stationary state and one stable limit cycle and (iii) one stable stationary state, one unstable limit cycle and one stable limit cycle. The third case of two limit cycles is of great importance for the explanation of patchiness initiation according to Dubois and Closset [1]. They show that if the grazing function $k(x)$ takes the form

$$
k(x)=\left\{\begin{array}{lll}
k_{2} x, & \text { if } & x \leq \tau \\
k_{2} \tau, & \text { if } & x>\tau
\end{array}\right.
$$

then the system has two limit cycles. Here $\tau$ represents the phyloplankton concentration at which saturation occurs, so $\tau$ is a threshold under which the grazing is linear and over which the grazing is constant. Under the threshold fluctuations are damped and over the threshold fluctuations are amplified initiating the emergence of new time and space patterns.

Furthermore $\tau, k_{1}, k_{2}$ and $k_{3}$ are positive constants while $\alpha \geq 0$ and $\beta \geq 0$. The system ( 1 and 2 ) takes on the properties of a typical Lotka-Volterra predator-prey system, where logistic growth of the prey species $x(t)$ is reflected by the $-k_{1} \alpha x^{2}$ term.

Dubois and Closset [1] use the system (1 and 2) to describe the patchiness effect of a plankton wave which is applicable in the field of marine biology. However, since it is in essence a Lotka-Volterra system subjected to piecewise conditions the system can likely be applied to other applications displaying predator-prey interactions.

When $x \leq \tau$, the system is governed by a linear predation rate where interaction is directly proportional to the availability of prey, but when $x>\tau$, the interaction between predator and prey describes a special case of the so-called Holling Type predation pattern, where predation is determined by the relative "fullness" of the predator in combination with emigration and immigration patterns of the prey species.

Dubois and Closset [1] found evidence on computer that System (1) have two limit cycles and the paper by Yongtai and Li [2] does a qualitative analysis to confirm Dubois and Closset's [1] conclusion of their existence. The analysis of Yongtai and Li [2] is cryptically written with three theorems and three lemmas as well as their proofs condensed to fill less than two pages. The paper has evidently not been language edited and therefore makes reading and comprehension difficult. For example, in the abstract of their paper the equation 
$\dot{x}=k_{1}(x-\alpha x)-k(x) y$ is incorrect. Further mistakes are subsequently pointed out in our paper. An undergraduate student (or even a graduate student) in mathematics would find it near impossible to make sense of the paper. A student in biology would most likely not be able to digest the paper at all, which has its roots in marine biology.

The purpose of this paper is firstly to present the results of the Yongtai and Li [2] paper in such a way that students from both a mathematical and biological background can work through it with fair ease. Graphical representations are generously presented to further the reader's understanding.

\section{Discussion}

First consider the quadratic population model given by

$$
\begin{aligned}
& \frac{d x}{d t}=x\left(a_{0}+a_{1} x+a_{2} y\right) \\
& \frac{d y}{d t}=y\left(b_{0}+b_{1} x+b_{2} y\right)
\end{aligned}
$$

where $a_{i}$ and $b_{i}$ are real constants $(i=0,1,2)$. This model has been well investigated by amongst others: Bautin [7, 8], Coppel [9], Coleman [10] and Van der Vaart [11]. In the latter, Van der Vaart confirms that this model can never have limit cycles, and finds conditions for the equilibrium point in the population quadrant to be a center, thereby resulting in a family of closed curves.

System (1) is an example of System (3), with the difference that it is subjected to piece-wise conditions.

For $x \leq \tau$, System (1) results in a typical predator-prey system

$$
\begin{aligned}
& \frac{d x}{d t}=k_{1} x-k_{1} \alpha x^{2}-k_{2} x y \\
& \frac{d y}{d t}=-k_{3} y+\beta k_{2} x y
\end{aligned}
$$

and for $x>\tau$,

$$
\begin{aligned}
& \frac{d x}{d t}=k_{1} x-k_{1} \alpha x^{2}-k_{2} \tau y \\
& \frac{d y}{d t}=-k_{3} y+\beta k_{2} \tau y
\end{aligned}
$$

where the constant $\tau>0$ acts as a prey population concentration threshold that will change the nature of the predator-prey interaction. The functions that make up System (1) are piecewise continuous and of exponential order, hence the existence and uniqueness of solutions can be confirmed [12]. Neither of the two Systems (A) or (B), considered individually, have limit cycles because they are both of the form of System (3) (Van der Vaart [11]). 
System (A), with $x \leq \tau$, has three equilibrium points, namely

$$
S_{1}=(0 ; 0), S_{2}=\left(\frac{1}{\alpha} ; 0\right) \text { and } S_{3}=\left(\frac{k_{3}}{\beta k_{2}} ; \frac{k_{1}}{k_{2}}\left(1-\alpha \frac{k_{3}}{\beta k_{2}}\right)\right)
$$

and System (B), with $x>\tau$, has two equilibrium points, $S_{1}=(0 ; 0)$ and $S_{2}=\left(\frac{1}{\alpha} ; 0\right)$.

Note that there is a typographical error in the Yongtai and Li [2] paper in the writing of $S_{3}$.

It should also be noted that $S_{3}$ is physically relevant if and only if $\alpha \frac{k_{3}}{\beta k_{2}}<1$.

Equilibrium points $S_{1}$ and $S_{2}$ predict extinction of one or both species and naturally neither is surrounded by closed curves contained in the population quadrant, which is a prerequisite for the existence of a limit cycle in population models. Equilibrium point $S_{3}$ is therefore the only likely candidate as equilibrium point to be surrounded by a closed periodic trajectory.

If $\frac{k_{3}}{\beta k_{2}} \geq \tau$, for both System (A) and (B), it follows that $\frac{d y}{d t} \leq 0$ for all $(x, y)$ in the first quadrant, and by the Bendixson negative criterion [12] no limit cycles are possible. This was stated as Theorem 1 in the Yongtai and Li [2] paper, with no reference to the Bendixson criteria which might make it more difficult to understand how they reached their conclusion of no limit cycles being possible.

So assume that $\frac{k_{3}}{\beta k_{2}}<\tau$. To further investigate the properties of $S_{3}=\left(\frac{k_{3}}{\beta k_{2}} ; \frac{k_{1}}{k_{2}}\left(1-\alpha \frac{k_{3}}{\beta k_{2}}\right)\right)$ first consider the case when $\alpha=0$. This case was dealt with as Lemma 2 in the Yongtai and $\mathrm{Li}$ [2] paper. The explanation in this paper gives more detail which can assist in understanding.

Special Case: $\alpha=0$

System (1) now becomes

with

$$
\begin{aligned}
& \frac{d x}{d t}=k_{1} x-k(x) y \\
& \frac{d y}{d t}=-k_{3} y+\beta k(x) y
\end{aligned}
$$

simplifying, for $x \leq \tau$, to

$$
k(x)=\left\{\begin{array}{lll}
k_{2} x, & \text { if } & x \leq \tau \\
k_{2} \tau, & \text { if } & x>\tau
\end{array}\right.
$$




$$
\begin{aligned}
& \frac{d x}{d t}=k_{1} x-k_{2} x y \\
& \frac{d y}{d t}=-k_{3} y+\beta k_{2} x y
\end{aligned}
$$

and for $x>\tau$ to

$$
\begin{aligned}
& \frac{d x}{d t}=k_{1} x-k_{2} \tau y \\
& \frac{d y}{d t}=-k_{3} y+\beta k_{2} \tau y
\end{aligned}
$$

System $\left(\mathrm{A}^{*}\right)$ has two equilibrium points $S_{1}=(0,0)$, which is a saddle, and $S_{3} *=\left(\frac{k_{3}}{\beta k_{2}}, \frac{k_{1}}{k_{2}}\right)$ a center, lying in the interior of the first quadrant. By taking the ratio of $\frac{d y}{d t}$ to $\frac{d x}{d t}$, System $\left(\mathrm{A}^{*}\right)$ is reduced to a single separable differential equation. The solutions to this system are closed periodic solutions defined by $k_{1} \ln y-k_{2} y+k_{3} \ln x-\beta k_{2} x=c$, where $c$ is a constant of integration. Therefore $S_{3} *$ is a center surrounded by infinitely many steady states, each depending on its unique initial value $\left(x_{0}, y_{0}\right)$.

The solution to System ( $\left.\mathrm{B}^{*}\right)$ is $y=\frac{k_{1}+k_{3}-\beta k_{2} \tau}{k_{2} \tau} x-c_{1} \frac{e^{k_{1} t}\left(k_{1}+k_{3}-\beta k_{2} \tau\right)}{k_{2} \tau}$ so for $c_{1}=0$ the system has a straight line solution, say $L_{1}$, given by $y=\frac{k_{1}+k_{3}-\beta k_{2} \tau}{k_{2} \tau} x$ that passes through the origin. If we assume that $k_{1}+k_{3}-\beta k_{2} \tau>0$ then $L_{1}$ has a positive gradient.

Now consider the combined solutions of Systems $\left(\mathrm{A}^{*}\right)$ and $\left(\mathrm{B}^{*}\right)$, subject to the piecewise defined conditions. When the $x$-values defining the trajectory become greater than $\tau$, the trajectory will intersect the vertical line $x=\tau$, and the characteristics of System ( $\left.\mathrm{B}^{*}\right)$ will then govern the future path of the trajectory. The vector field of System (4) is shown in Figure 1 on which the demarcation brought about by $x=\tau$ is clear. 


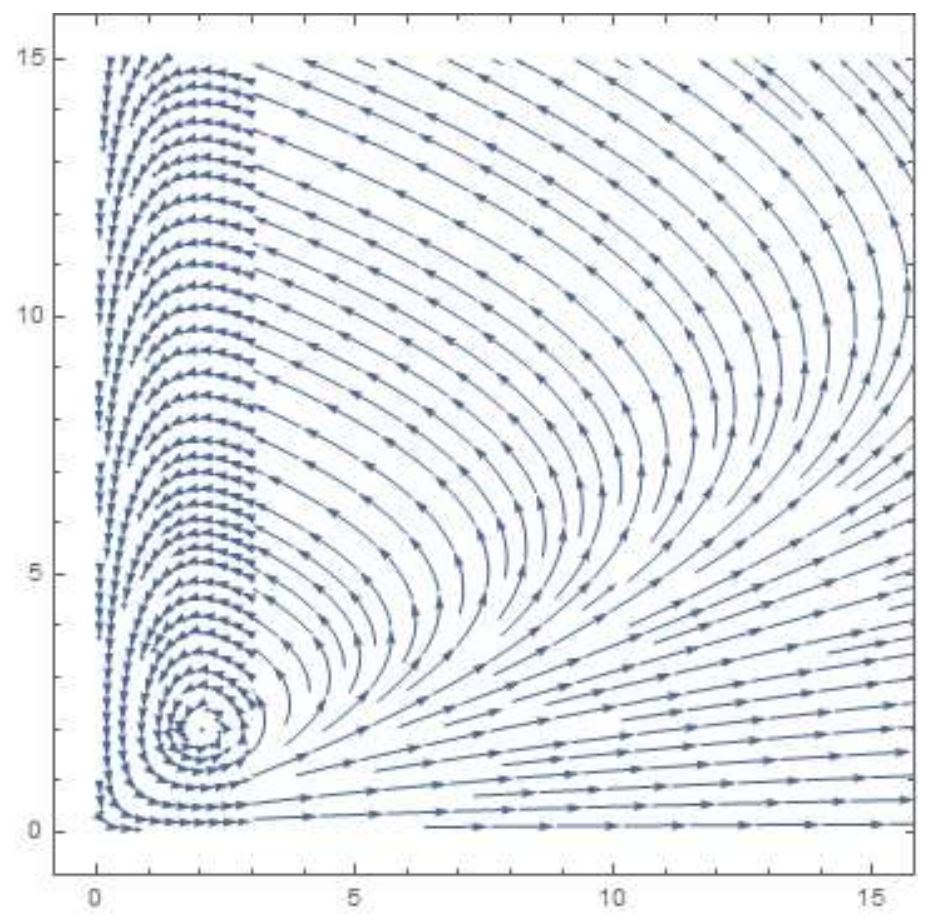

Figure 1: The vector field of System (4).

Some of the closed curve solutions of System (A*), as shown in Figure 2, must intersect the straight line $x=\tau$ in two points. We consider one of these closed curve solutions and denote the points of intersection as $P_{0}\left(\tau, y_{0}\right)$ and $P_{1}\left(\tau, y_{1}\right)$.

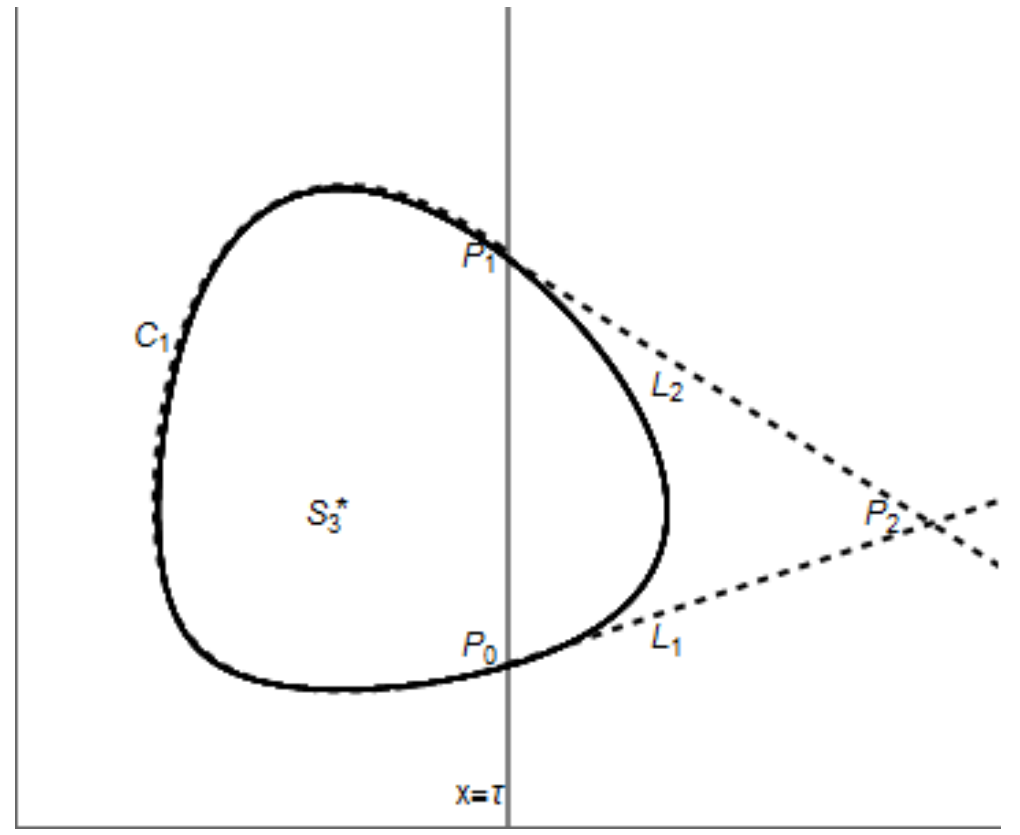

Figure 2: Closed curve intersecting $x=\tau$ in $P_{0}\left(\tau, y_{0}\right)$ and $P_{1}\left(\tau, y_{1}\right)$ 
We choose initial value $P_{0}\left(\tau, y_{0}\right)$ where $y_{0}=\frac{k_{1}+k_{3}-\beta k_{2} \tau}{k_{2}}$ so that it is situated on the straight line $L_{1}$ which is a solution to System $\left(\mathrm{B}^{*}\right)$. Note that there is a typographical error in the Yongtai and $\mathrm{Li}[2]$ paper in the typing of $y_{0}$.

It would therefore be possible to construct at least one straight line segment, say $L_{2}$, with negative gradient, connecting $P_{1}\left(\tau, y_{1}\right)$ to some point on the solution $L_{1}$ of System $\left(\mathrm{B}^{*}\right)$, say $P_{2}$, for which the vector field of System (4) will depart in an upward direction as shown in Figure 3.

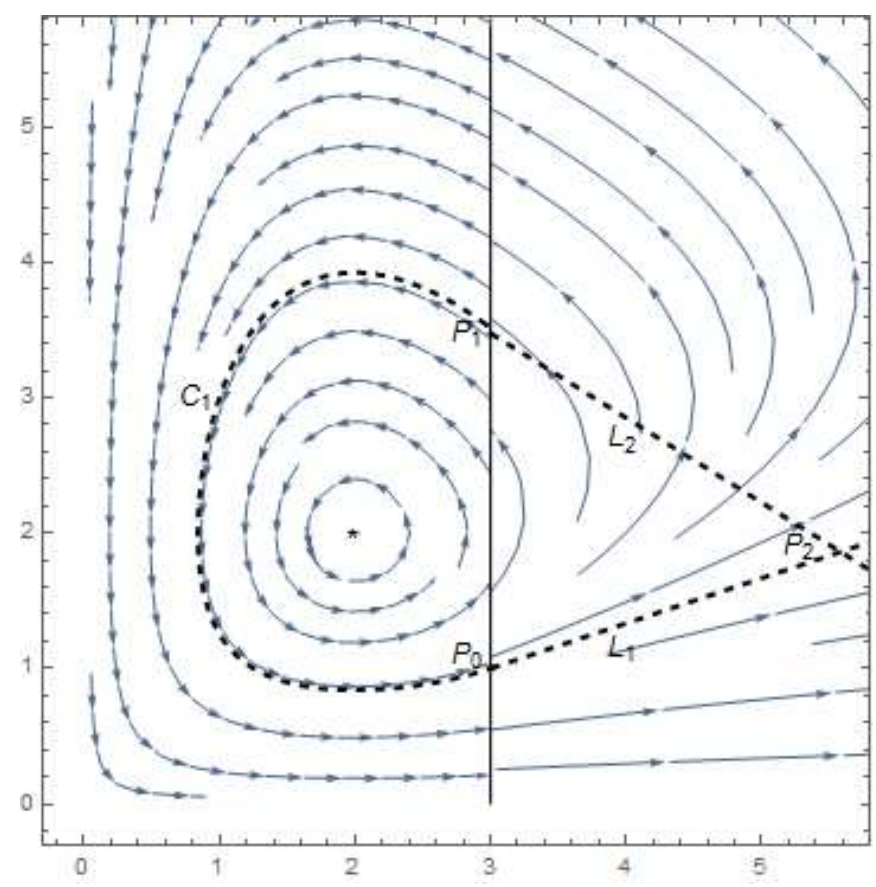

Figure 3: Vector field of System (4)

For System (4) there now exists a simple closed region defined by curve $C=\overline{P_{1} P_{0}} \cup \overline{P_{0} P_{2}} \cup \overline{P_{2} P_{1}}$ where $\overline{P_{1} P_{0}}$ is the solution curve segment $C_{1}$ of System $(\mathrm{A} *), \overline{P_{0} P_{2}}$ is the solution line segment $L_{1}$ of System $\left(\mathrm{B}^{*}\right)$, and $\overline{P_{2} P_{1}}$ is the straight line segment $L_{2}$ on which the vector field of System (4) departs in an upward direction. There are several mistakes in the Yongtai and $\mathrm{Li}$ [2] paper that makes visualization of the region difficult. This region is illustrated in Figure 4. This figure serves as a valuable illustration and was not included in the paper by Yongtai and $\mathrm{Li}$ [2]. The parameter values used to generate the figures are as follows: $k_{1}=2, k_{2}=1, k_{3}=2, \beta=1$ and $\tau=3$. So $S_{3}^{*}=(2,2)$ is a center if $x \leq 3$ and $L_{1}$ is the straight line solution if $x>3$. 


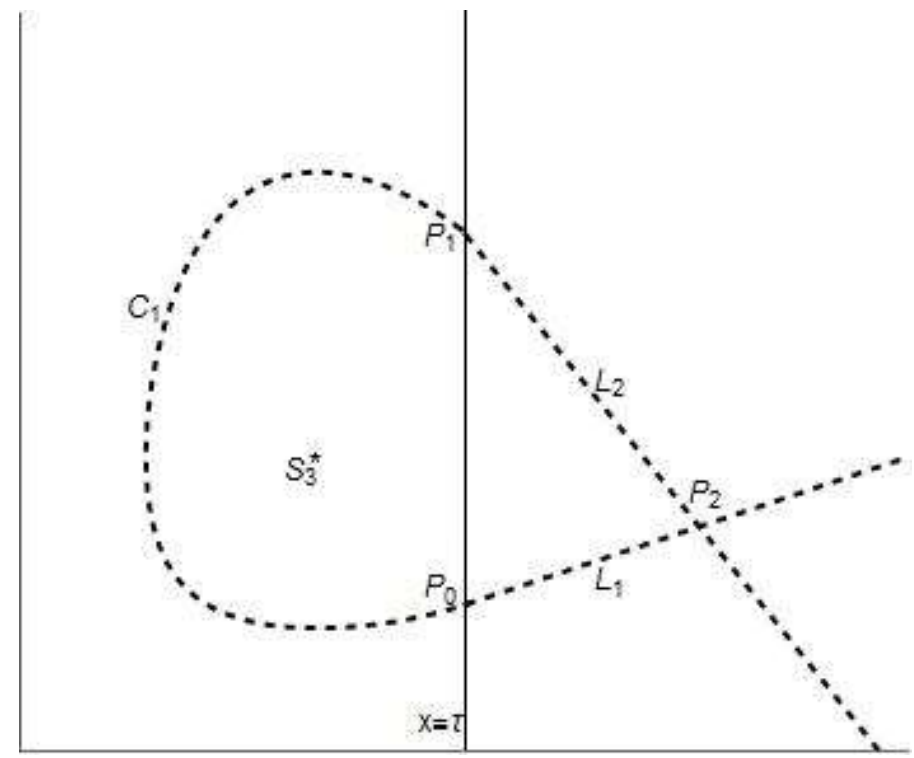

Figure 4: The curve $C$ defined for System (4)

The region enclosed by $C$ is such that, for System (4), there is no vector field entering the region, implicating that all solution curves generated outside this region in positive time will remain outside the region. Any solution curve initiated, in negative time, from inside the region $C$ will be attracted by the limit cycle as is the case when initiated from outside the region $\mathrm{C}$ in negative time as is shown in Figure 5.

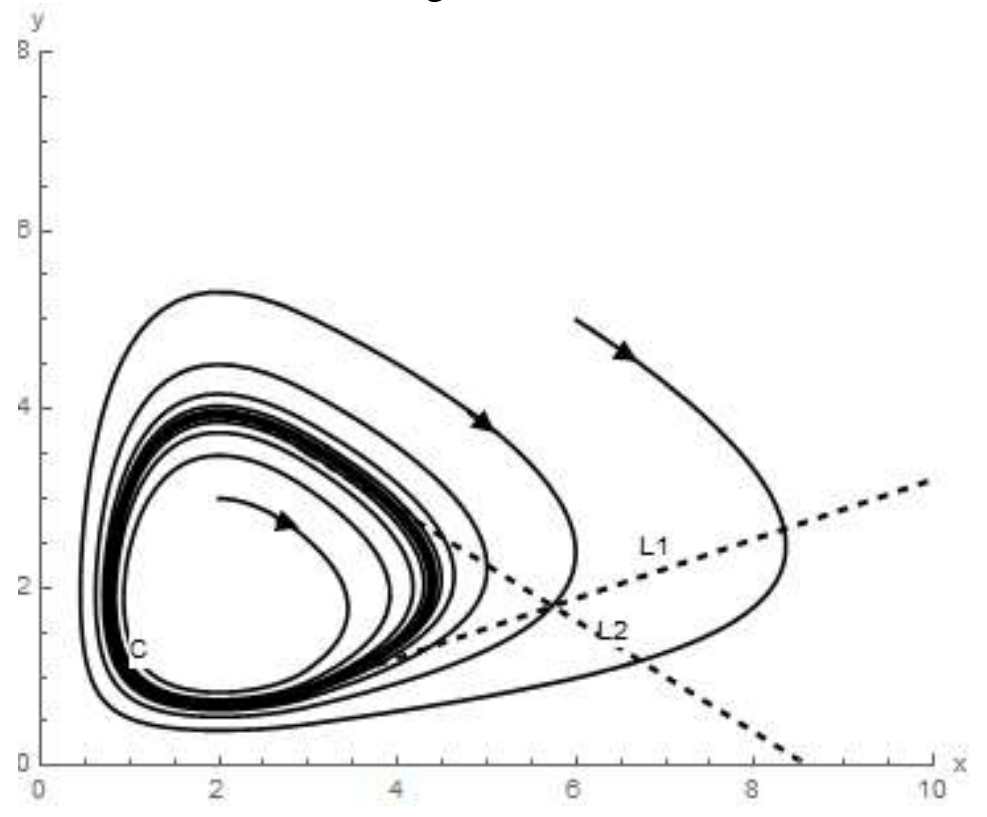

Figure 5: Solution curves attracted to the limit cycle in negative time.

Case when $0<\alpha<<1$ (Theorem 2 in the Yongtai and Li [2] paper.)

By the principle of continuous dependence of solutions on parameters for $0<\alpha<<1$, System (1) still has a closed curve $C$ on which no vector field of System (1) will enter the interior. The general continuous dependence of solutions on parameters allows us to derive qualitative 
statements regarding nonlinear systems [13] as long as we stay sufficiently close to $\alpha=0$. Thus for $\frac{k_{3}}{\beta k_{2}}<\tau$ and $k_{1}+k_{3}-\beta k_{2} \tau>0$. System (1) has a limit cycle with $S_{3}$ as equilibrium point.

Furthermore, for System (1) and for some large value of $h$, that is $h>>1$, there exists a straight line, say $L_{3}$, defined by $y+\beta x=h$ on which there is no vector field of System (1) tending upwards from it. This is motivated as follows:

Consider

$$
\begin{aligned}
\frac{d L_{3}}{d t}=\frac{d}{d t}(y+\beta x) & =\frac{d y}{d t}+\beta \frac{d x}{d t} \\
& =\left(-k_{3}+\beta k(x)\right) y+\beta\left(k_{1} x(1-\alpha x)-k(x) y\right) .
\end{aligned}
$$

But $y=-\beta x+h$, therefore

$$
\begin{aligned}
\frac{d L_{3}}{d t} & =\left(-k_{3}\right)(h-\beta x)+\beta\left(k_{1} x(1-\alpha x)\right) \\
& =x \beta k_{1}-h k_{3}+x \beta k_{3}-x^{2} \alpha \beta k_{1} \\
& =-\left(\alpha \beta k_{1} x^{2}-\beta\left(k_{1}+k_{3}\right) x+h k_{3}\right)
\end{aligned}
$$

Thus, for some large value of $h$, the gradient $\frac{d L_{3}}{d t}<0$ for all $(x, y)$ so that the vector field for System (1) departs upwards on $L_{2}$ and anticlockwise away from $L_{3}$. We have provided more detail than in the Yongtai and $\mathrm{Li}$ [2] paper presented there as Lemma 3. So we now have two regions: one inside the curve $C$, say $R_{1}$ and one outside the curve $C$ but below $L_{3}$, say $R_{2}$. This is depicted in Figure 6.

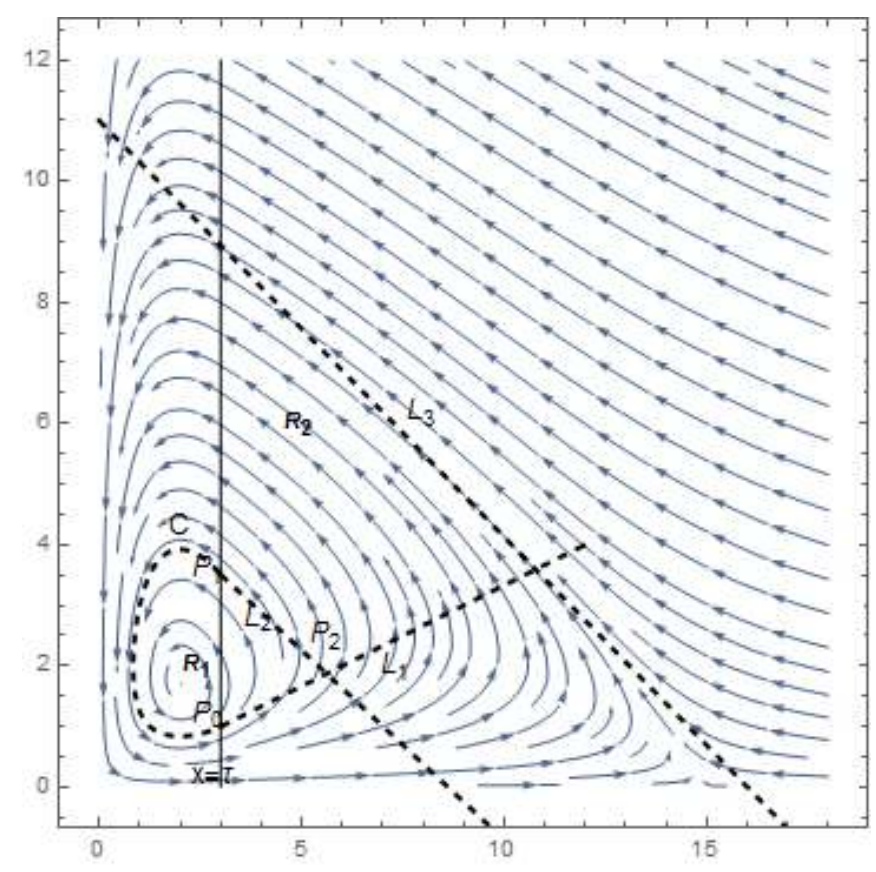


Figure 6: Vector fields of System (1) showing the regions $R_{1}$ and $R_{2}$.

This results in a second limit cycle being formed. This result is presented in the Yongtai and Li [2] paper as Theorem 3. The illustration provided in Figure 6 should help to understand how the second limit cycle is formed.

Neither Dubois and Closset [1] or Yongtai and Li [2] included examples in their papers.

We illustrate the existence of the two limit cycles by the following example.

\section{Example}

Parameter values are chosen to satisfy the conditions dealt with in this paper. It has no bearing on any specific predator-prey interactions and the novelty lies within the discovery of the two limit cycles.

Choose $k_{1}=2 ; k_{2}=1 ; k_{3}=2 ; \tau=3 ; \beta=1$ and $\alpha=1 / 15 \quad$ so that $\quad k_{3}-\beta k_{2} \tau<0$ and $k_{1}+k_{3}-\beta k_{2} \tau>0$. Equilibrium point $S_{3}$ is now a stable spiral point as shown in Figure 7. All trajectories initiated in positive time from within the region $R_{1}$ spirals towards the equilibrium point $S_{3}$ (in this case $\left(2, \frac{26}{15}\right)$ ). In negative time the trajectories will be attracted to the limit cycle as also shown in Figure 5 and 7.

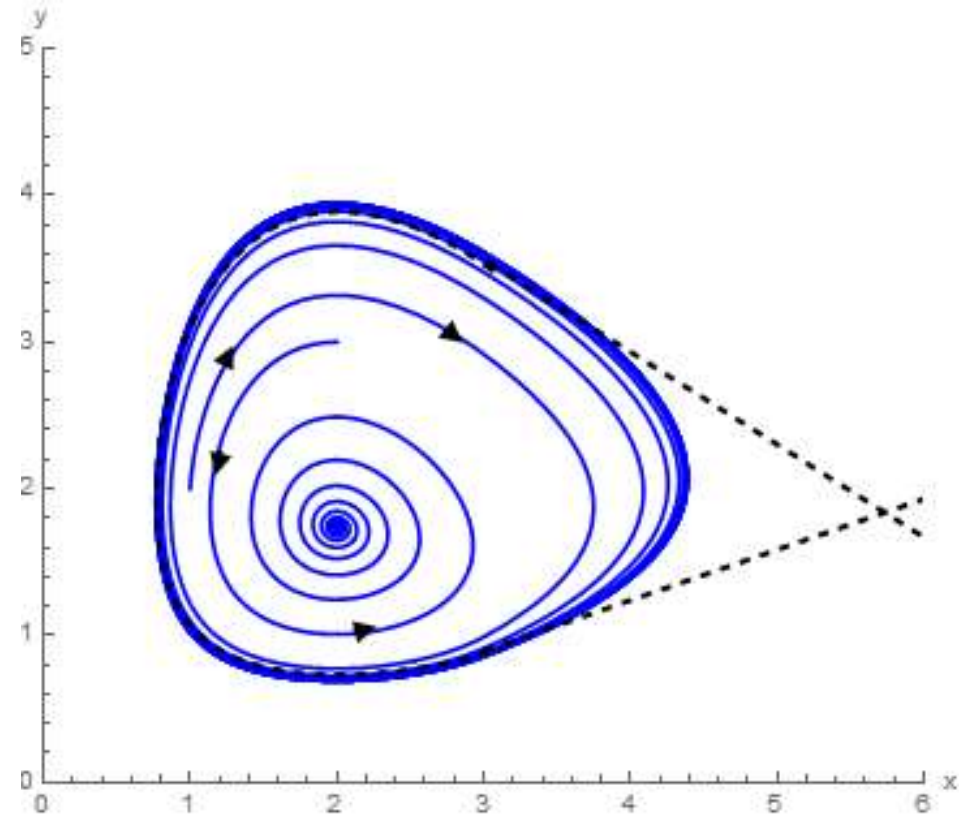

Figure 7: If $\alpha=\frac{1}{15}$ the equilibrium point $S_{3}=\left(2, \frac{26}{15}\right)$ is a stable spiral point in positive time.

For any initial value, whether taken from $R_{1}$ or $R_{2}$ the solution of System (1) results in a limit cycle in negative time $(-100 \leq t \leq 0)$. Figure 8 a shows a trajectory tending to a limit cycle when the initial value $(2,3)$ taken from $R_{1}$ is used, while Figure $8 \mathrm{~b}$ is the result when initial value $(4,4)$ taken from $R_{2}$ is used. 


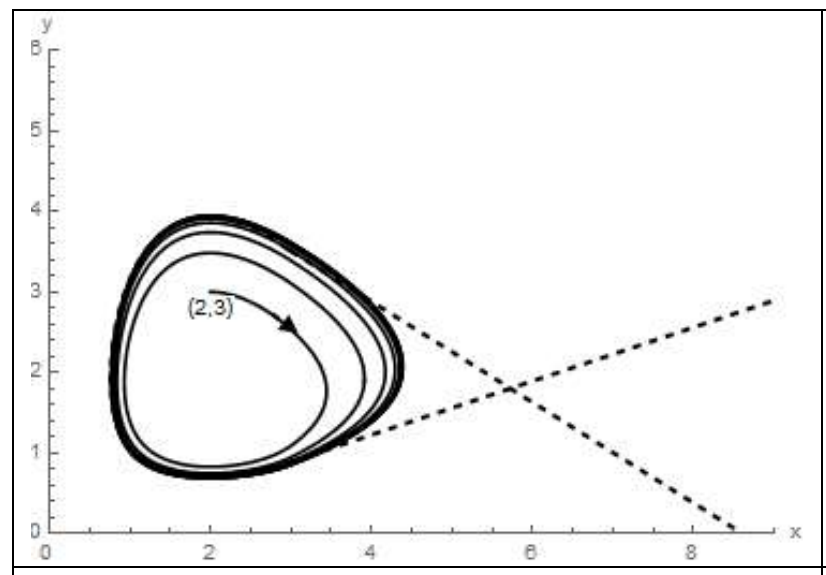

Figure 8a: A trajectory initiated at $(2,3)$ on the inside of curve $\mathrm{C}$ tends to a limit cycle.

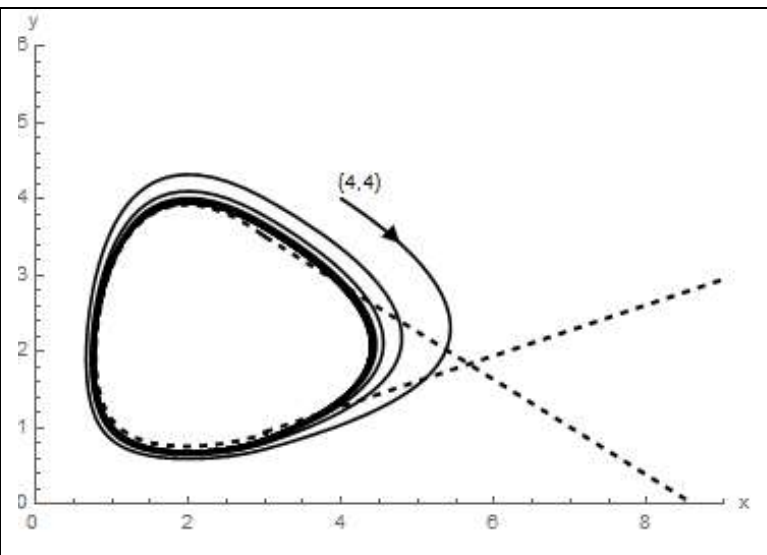

Figure 8b: A trajectory initiated at $(4,4)$ on the outside of curve $C$ tends to the same limit cycle

For any initial value taken from $R_{2}$ or above $L_{3}$, the solutions of System (1) will form a second limit cycle in positive time $(0 \leq t \leq 100)$. The second limit cycle is generated with initial value $(5,4)$ taken from $R_{2}$ (Figure 9a) and $(10,8)$ from the region above $L_{3}$ (Figure 9 b) respectively.

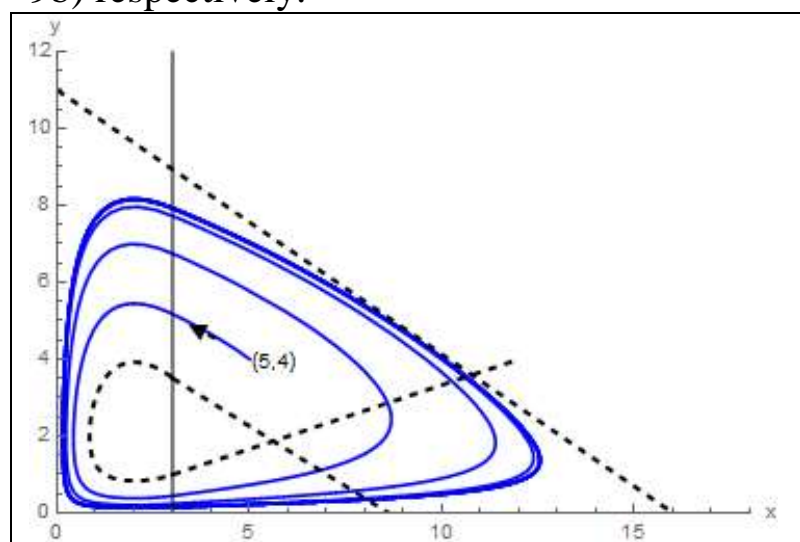

Figure 9a: Limit cycle formed with initial value $(5,4)$ taken from $R_{2}$

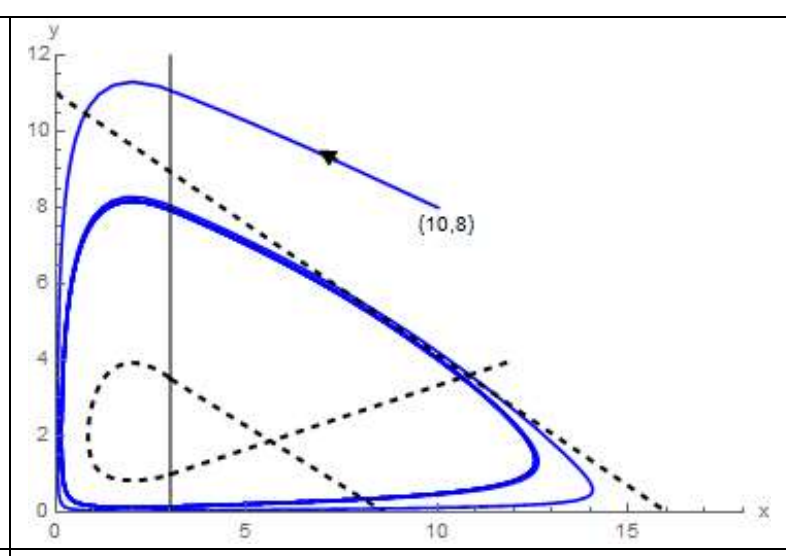

Figure 9b: Limit cycle formed with initial value $(10,8)$ taken from above $L_{3}$.

In Figure 10 initial values were taken from all three regions, $R_{1}, R_{2}$ and the region above $L_{3}$, describing the behavior of the trajectories in positive time. 


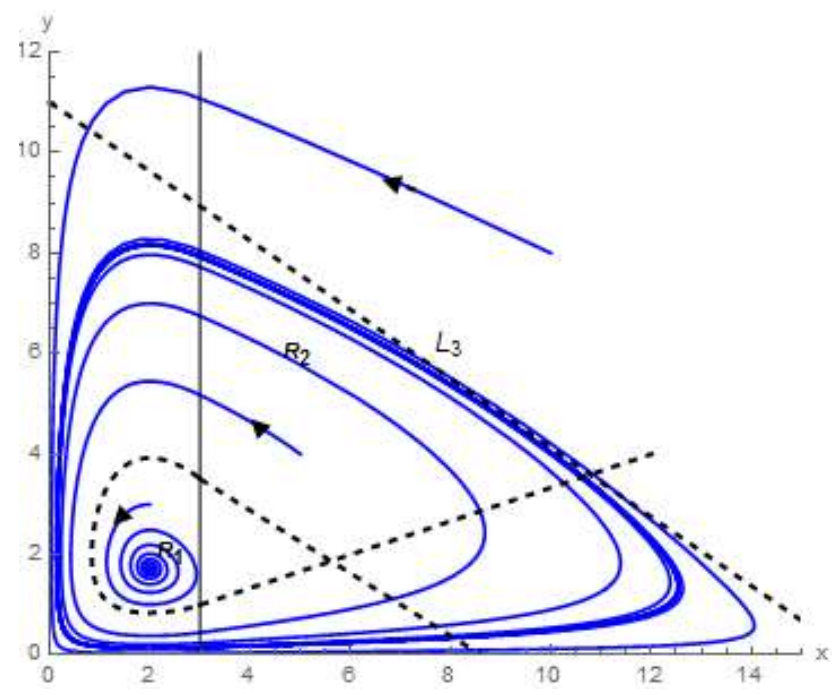

Figure 10: Trajectories of System (1) in positive time.

Finally, in Figures 11a and 11b the formation of the two limit cycles formed by System (1) is shown.

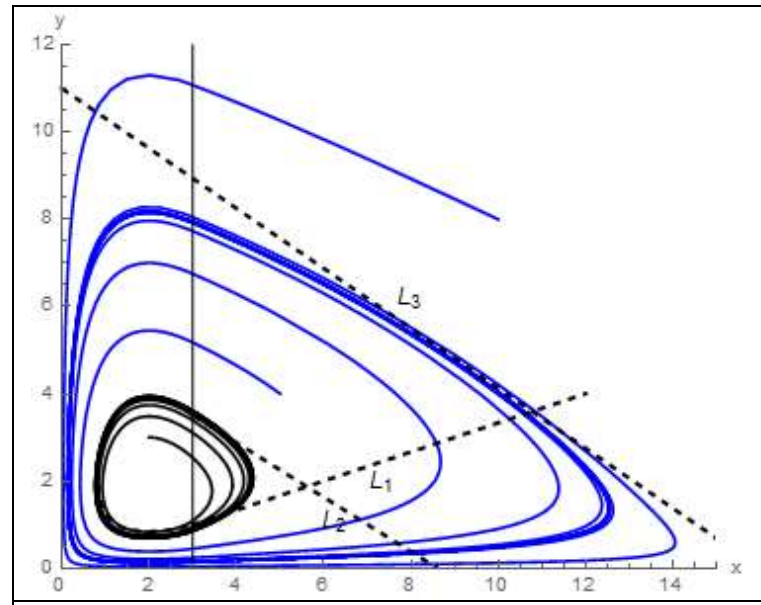

Figure 11a: Two limit cycles formed by generating trajectories in negative and positive time, respectively.

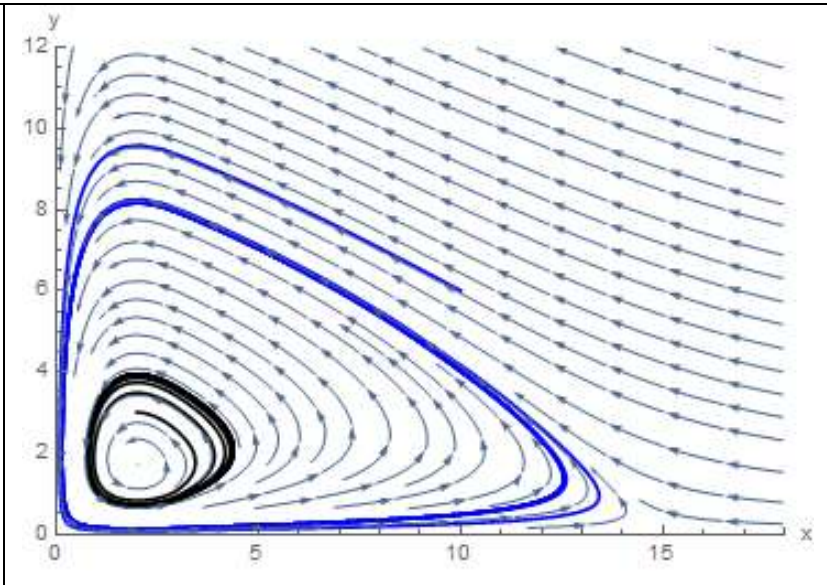

Figure 11b: The vector field of System (1)

\section{Further studies}

As long as $0<\alpha<<1$, the closed curve $C$ will exist and therefore no vector field of System (1) will enter the region $R_{1}$. This begs the question: how "small" must $\alpha$ be? In our example the value of $\alpha=1 / 15$ was chosen arbitrarily and the existence of two limit cycles was evident.

With some experimentation it seems that for $\alpha=1 / 13$ both limit cycles still exist. However, for $\alpha=1 / 12$, the results change drastically. Running $t$ from 0 to 500 with initial value $(10,6)$ 
the trajectory "passes" through the "limit cycle" spiraling in towards the stable equilibrium as shown in Figure 12.

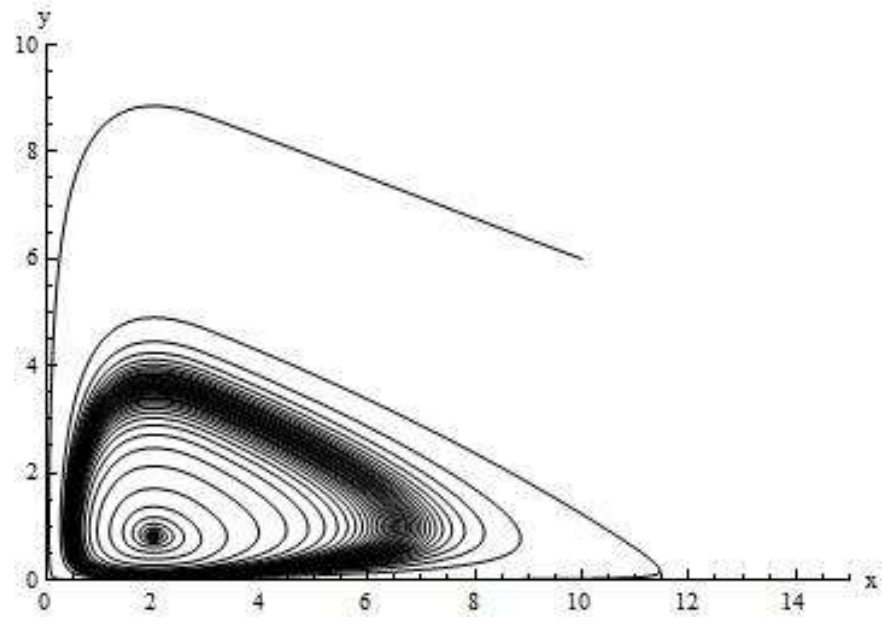

Figure 12: If $\alpha=1 / 12$ then the equilibrium becomes a stable spiral.

Further experimentation on the value of $\alpha$ may offer a valuable route of exploration.

Furthermore, Dubois and Closset [1] found evidence on computer that System (1) have two limit cycles and the paper by Yongtai and $\mathrm{Li}$ [2] does a qualitative study and proves that this is indeed the case under certain conditions namely $k_{3}-\beta k_{2} \tau<0$ and $k_{1}+k_{3}-\beta k_{2} \tau>0$. We know that no limit cycle is possible should $k_{3}-\beta k_{2} \tau \geq 0$ but what if $k_{3}-\beta k_{2} \tau<0$ and $k_{1}+k_{3}-\beta k_{2} \tau<0$. Initial experimentation indicates that we still have two limit cycles, but this has not been investigated further in this article.

\section{Conclusion}

By unpacking the model an example of basic research is given that should be of value to a young academic interested in biomathematics. The knowledge and expertise gained through critical reading of an article requiring knowledge from more than one discipline should be invaluable. Aspects of modeling and ecology are touched upon as well as technological prowess, differential equations and vector analysis. The exposition presented here should be within the grasp of either someone schooled in biology with basic understanding of differential equations or someone schooled in mathematics and venturing into applications in modeling and biology.

The untangled mathematics mechanism of a model dating from the 1980's combined with rich illustrations, presented here, moves the dated cryptic exposition of a distinct model into the current paradigm of technology use in advancing the field of biomathematics.

\section{References}

[1] Dubois DM, Closset PL. Patchiness in primary and secondary production in the Southern Bight: a mathematical theory, $10^{\text {th }}$ European Symposium on Marine Biology, Ostend, Belgium, Sept. 17-23, 1975; 2:211-229.

[2] Yongtai R, Li H. The Predator-Prey Model with Two Limit Cycles, Acta Mathematicae Applicatae Sinica. 1989; 5: 30 - 32. 
[3] Eager EA, Pierce J, Barlow P. Math Bio or Biomath? Flipping the mathematical biology classroom. Letters in Biomathematics. 2014;2:139-155.

[4] Van der Hoff Q. Interdisciplinary education - a predator-prey model for developing a skill set in mathematics, biology and technology, International Journal of Mathematical Education in Science and Technology, 2017;48:6, 928-938.

[5] Feser J, Vasaly H, Herrera J. On the edge of Mathematics and Biology Integration: Improving Quantitative Skills in Undergraduate Biology Education. CBE-Life Sciences Education. 2013;12:124-128.

[6] Wolfram S. The Mathematica Book, 3rd ed. Wolfram Media /Cambridge University Press, New York. 1999.

[7] Bautin NN. On the number of limit cycles which appear with the variation of coefficients from an equilibrium position of focus or center type (R). Mat. Sb. 1952;30(72):181-196. Amer. Math. Sot. Transl. 1954;100.

[8] Bautin NN. On periodic solutions of a system of differential equations (R). Prikl. Mat. Meh. 1954;18:128.4.

[9] Coppel A. A Survey of Quadratic Systems. Journal of Differential Equations 2. 1966; $293-304$.

[10] Braun M, Coleman CS, Drew DA. Differential Equations Models. Chapter 16, Quadratic Population Models: Almost never any cycles. 1978;229 - 248.

[11] Van der Vaart HR. Conditions for periodic solutions of Volterra differential systems. Bulletin of Mathematical Biology. 1978;40:133 - 160.

[12] Zill DG, Cullen MR. Advanced Engineering Mathematics. Second ed. Boston, Massachusetts: Jones and Bartlett Publishers. 2000.

[13] Megretski A. Dynamics of Nonlinear Systems. Department of Electrical Engineering and Computer Science, Massachusetts Institute of Technology. [Internet]. 2003 [cited 9 May 2017]. Available from https://ocw.mit.edu/courses/electrical-engineering-and-computer-science/6-243jdynamics-of-nonlinear-systems-fall-2003/lecture-notes/lec3_6243_2003.pdf 